\title{
Blue straggler populations beyond the Milky Way
}

\author{
Richard de Grijs ${ }^{1,2 *}$, Weijia Sun ${ }^{3,4}$, Chengyuan Li, ${ }^{1}$ Licai Deng ${ }^{4}$ \\ ${ }^{1}$ Department of Physics and Astronomy, Macquarie University, Balaclava Road, Sydney, NSW \\ 2109, Australia \\ *E-mail: richard.de-grijs@mq.edu.au \\ ${ }^{2}$ International Space Science Institute-Beijing, 1 Nanertiao, Zhonguancun, Hai Dian District, \\ Beijing 100190, China \\ ${ }^{3}$ Kavli Institute for Astronomy and Astrophysics, Peking University, Yi He Yuan Lu 5, Hai \\ Dian District, Beijing 100871, China \\ ${ }^{4}$ Key Laboratory for Optical Astronomy, National Astronomical Observatories, Chinese \\ Academy of Sciences, 20A Datun Road, Chaoyang District, Beijing 100012, China
}

\begin{abstract}
Although the formation of blue straggler stars (BSSs) is routinely attributed to stellar interactions in binary systems, the relative importance of the direct collision and slow(er) stellar coalescence formation channels is still poorly understood. We selected a sample of 24 Magellanic Cloud star clusters for which multi-passband Hubble Space Telescope images are available to address this outstanding question. We compiled a BSS database, containing both traditional and evolved BSSs. We found a robust correlation between the number of BSSs in a cluster's core and its core mass, $N_{\mathrm{BSS}, \text { core }} \propto M_{\text {core }}^{0.51 \pm 0.07}$, which supports the notion that BSS formation is linked to a population's binary fraction. At low stellar collision rates, the mass-normalised number of BSSs does not appear to depend on the collision rate, which implies that the coalescence-driven BSS formation channel dominates. Comparison with simulations suggests that stellar collisions contribute less than $20 \%$ to the total number of BSSs formed.
\end{abstract}

Keywords: blue stragglers - galaxies: star clusters - HertzsprungRussell and C-M diagrams - Magellanic Clouds.

\section{Blue straggler stars}

Blue straggler stars (BSSs) are among the most visible deviations from the once generally accepted 'simple stellar population' model of star clus- 
ters. In essence, this model assumes that star clusters were formed almost instantaneously from the same progenitor molecular cloud, so that the nascent stars have almost identical ages and the same chemical composition. Translated into colour-magnitude space, the Hertzprung-Russell diagrams of single-aged, single-metallicity star clusters would exhibit narrow stellar evolutionary features, including a tight main sequence and a well-defined, sharp main-sequence turn-off, in turn leading to narrow subgiant and red giant branches. BSSs, which are predominantly found at brighter magnitudes and bluer colours than the main-sequence turn-offs of their host clusters, do not fit well into this picture.

First discovered by Sandage (1953) in the outer regions of the old Galactic globular cluster (GC) M3, many decades of research effort has led to the realisation that BSS formation is intimately linked to the evolution of stellar binary systems. In essence, the primary and secondary components of the binary system evolve into a single object with the combined mass of its progenitor components to gain a new lease of life as an apparently rejuvenated BSS. The current consensus is that BSSs can form in one of two ways, that is, through direct collisions between two stars and through a slower process leading to stellar coalescence. In the former scenario, when two low-mass stars collide, they will form a gravitationally bound system that is subject to rotation to satisfy the conservation of angular momentum. As both stars merge, they release some of the collision products in the form of debris, which eventually disperses.

The resulting object is a more massive, rapidly rotating star that appears blue because the stellar atmospheres of the progenitor stars have been stirred up violently, allowing core materials to float up to the surface layers. The merged collision product gradually heats up under the effects of gravitational contraction and it eventually expands to become a red giantlike star. Magnetic braking causes a reduction in the star's rotation rate, allowing the star to shrink, heat up again, and eventually settle as a slowly rotating BSS. In the coalescence model, the resulting merger into a more massive star occurs on a much longer timescale. The merger product is a rapidly rotating BSS.

Observational evidence in apparent support of these two BSS formation channels was first provided by Ferraro et al. (2009), who showed that the colour-magnitude diagram of the core-collapse Galactic GC M30 exhibited two clearly separated 'sequences' of stars in the area of parameter space usually occupied by BSSs. The fainter, blue sequence appeared to be an extension of the cluster's single-star main sequence to brighter magnitudes (that is, including younger ages but offset by $\Delta V \sim-0.4$ mag with respect to the younger extension of the isochrone describing the GC's bulk stellar population), while the bottom envelope of the brighter, red swarm of data points coincided with the locus of the equal-mass binary sequence if extended to higher masses than those defining the main-sequence turn- 
off. The natural interpretation of these observations was that the blue BSS sequence had resulted from stellar collisions triggered by the cluster's core collapse, while the red main sequence and the stellar sample at brighter magnitudes may have formed through stellar coalescence (but see below). Note that this latter sample in Ferraro et al. (2009) observations did not occupy a single, well-defined red sequence, presumably because a fraction of the coalescence products had already undergone further evolution, and thus they would have started to move away from their birth environment in colour-magnitude space. Alternatively, some of those objects could simply be unresolved interacting binary systems involving non-main-sequence components that have yet to merge.

Further evidence provided by Ferraro et al. (2009) suggested that the collision products were mostly found in the GC's core environment, while the coalescence products occupied larger clustercentric radii. Similar results were reported by Li et al. (2013) for the Large Magellanic Cloud (LMC) GC Hodge 11, although their results were less clear-cut. At the larger distance of the LMC, of order $50 \mathrm{kpc}$, the effects of (back- or foreground) field contamination and stellar crowding could significantly affect the quality and reliability of photometric observations of dense star clusters in their outer and core regions, respectively.

\section{Star clusters in the Magellanic Clouds}

Nevertheless, the Magellanic Clouds are better environments to study the evolution of dense, massive star clusters than the Milk Way, for a number of important reasons: (i) star clusters in the Magellanic Clouds cover a large range in ages (by contrast, Milky Way GCs are almost uniformly older than 10 Gyr); (ii) given their far southern location in the sky, the Clouds are located far from the Galactic plane, and hence they are negligibly affected by foreground extinction (although a relatively small level of internal extinction still needs to be dealt with); and (iii) the set of massive star clusters in the Magellanic Clouds are less dense than their Galactic GC counterparts, thus counteracting the distance effects (i.e., stellar crowding) to some extent.

Despite these clear advantages of exploring the physical properties of Magellanic Cloud clusters, their large distances still require us to avail ourselves of high-resolution imaging observations with the Hubble Space Telescope (HST), particularly using the Advanced Camera for Surveys (ACS)/ Wide-Field Channel (WFC) or the Ultraviolet-Visible (UVIS) channel/WideField Camera-3 (WFC3), to resolve the bulk of their stellar populations. The resolution of $\sim 80$ milli-arcsec at optical wavelength attainable with these instruments translates to $\sim 0.02 \mathrm{pc}$ at the distance of the LMC. For our analysis we selected all intermediate-age (1-3 Gyr-old) and old ( 10 Gyrold) clusters in the Magellanic Clouds for which suitable HST observations 
were available. Young massive clusters were excluded, because the latter do not exhibit clear main-sequence turn-offs, which thus complicates the selection of BSSs. Application of these considerations resulted in a sample of 24 massive clusters (for details, see Sun et al. 2018).

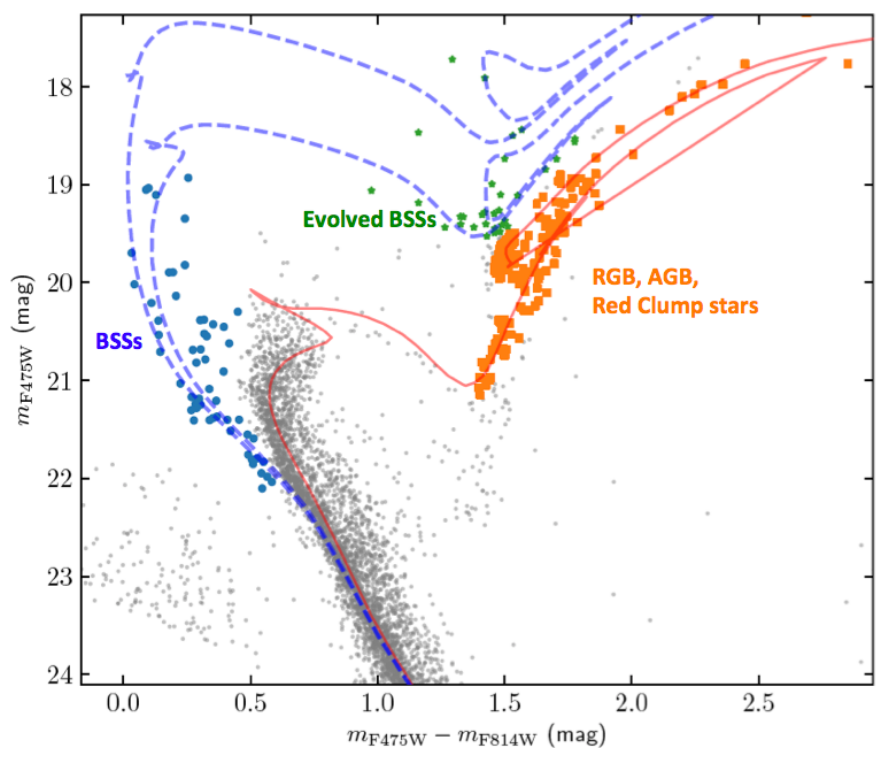

Figure 1: Colour-magnitude diagram of the LMC star cluster NGC 2173. BSSs, evolved BSSs, as well as red giant branch (RGB)/asymptotic giant branch (AGB)/red clump stars are marked with blue circles, green pentagons, and orange squares, respectively. The best-fitting PARSEC isochrones (Bressan et al. 2012) for the bulk stellar population (red solid line) and the BSS population (blue dashed line) are also shown. (Adapted from Sun et al. 2018.)

In a series of recent articles (Li et al. 2018; Sun et al. 2018), we realised that while the best-fitting isochrones representing the bulk stellar populations in our sample of Magellanic Cloud star clusters also matched the ridgelines of their red giant branches very well, many clusters exhibited significant numbers of stars blueward of the blue envelopes defined by the red giant branch ridgelines and well beyond the prevailing $3 \sigma$ observational uncertainties. In fact, isochrone fits to the cluster's BSS sequences appeared to adequately match those bluer red giant branch-like stars, which led us to suggest that the latter objects might be evolved BSSs: see Fig. 1. We carefully validated this idea on the basis of Monte Carlo simulations including the prevailing uncertainties, the number distributions, as well as their spatial distributions ( $\mathrm{Li}$ et al. 2018; Sun et al. 2018). Henceforth, in relation to each of our sample clusters, we will therefore refer to (i) our core BSS sample and (ii) our evolved BSS sample. BSSs are found in all of our clusters, with BSS numbers in their cores ranging from five to 70 . Evolved 
BSSs can be resolved in the intermediate-age clusters using optical filters, but they cannot be disentangled easily from the red clump in old clusters.

Following Knigge et al. (2009) and Leigh et al. (2013), we explored whether our Magellanic Cloud BSS numbers, $N_{\mathrm{BSS}}$, also correlated with the core mass, $M_{\text {core }}$, of their host clusters. Knigge et al. (2009) and Leigh et al. (2013) reported a relationship of the form

$$
N_{\text {BSS,core }} \propto M_{\text {core }}^{\alpha} ; \alpha \sim 0.5
$$

for samples of 57 and 30 additional Galactic GCs, respectively. Our 24 LMC clusters, on the other hand, yielded a significantly steeper proportionality,

$$
N_{\text {BSS }, \text { core }} \propto M_{\text {core }}^{\alpha_{\mathrm{c}}} ; \alpha_{\mathrm{c}}=0.66 \pm 0.07
$$

and a Spearman coefficient of $\rho_{\mathrm{S}}=0.84$ (Sun et al. 2018). If we include the 'evolved BSSs,' however, the power-law index of this latter proportionality becomes $\alpha_{\mathrm{c}+\mathrm{e}}=0.51 \pm 0.07$ (and $\rho_{\mathrm{S}}=0.80$ ), which implies a robust correlation that is indeed very close to the previously published Galactic GC results.

Knigge et al. (2009) suggested that the 'sublinear' relation between the number of BSS stars in a cluster and its core mass could be explained as the result of binary interactions if the binary fraction, $f_{\text {bin }}$, also depends on the core mass (Milone et al. 2012),

$$
f_{\text {bin }} \propto M_{\text {core }}^{-0.5} .
$$

However, Leigh et al. (2013) found that including the numbers of binary stars in the cores of their sample of 30 additional Galactic GCs did not strengthen the correlation, either because of the empirical uncertainties affecting the derived binary fractions or owing to the correlation's intrinsic dispersion; the resulting degradation of the correlation further complicated efforts to understand the BSSs' origins.

Therefore, we explored whether the numbers of BSSs, both in the cluster cores and including the evolved counterparts, might be correlated with the clusters' representative (annual) stellar collision rates: see Fig. 2. We normalised both quantities by the clusters' core masses to reduce any effects owing to a dependence of the collision rates on the core masses of our clusters. It is clear that in low collision-rate clusters, the collision rates do not depend on core mass. At high collision rates, on the other hand, BSS formation appears to be suppressed. We suspected that this could be evidence of the importance of binary-mediated BSS formation.

Figure 2 also includes the results of the numerical simulations performed by Chatterjee et al. (2013). The simulation results shown in, respectively, the left- and right-hand panels highlight BSSs formed through coalescence of binary systems and direct collisions, for different initial binary fractions. The 

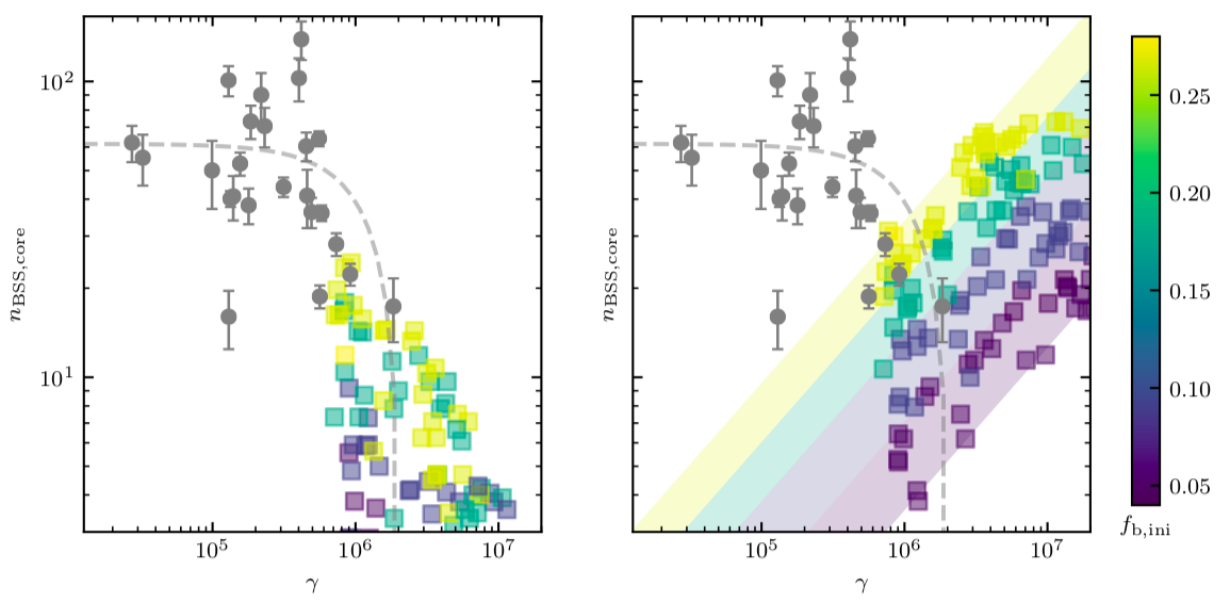

Figure 2: Mass-normalised number of BSSs in the core $\left(n_{\mathrm{BSS}, \mathrm{core}}\right)$ as a function of the mass-normalised collision parameter, $\gamma$ (single star collisions only). Magellanic Cloud clusters are shown as the grey data points. The grey dashed line represents the best-fitting model of the form $n=C+a \gamma^{b}$. The simulation of Chatterjee et al. (2013) is overplotted. BSSs formed through the binary and collision channels in the simulation are shown in, respectively, the left- and right-hand panels as coloured squares, for different initial binary fractions $\left(f_{\mathrm{b}, \text { ini }}\right)$. The shaded regions encompass the possible contributions from collisions in lower collision-rate environments. (Source: Sun et al. 2018.)

left-hand panel shows that the mass-normalised number of BSSs generated from primordial binaries in the Chatterjee et al. (2013) simulations declines toward higher collision rates, as observed in our Magellanic Cloud cluster sample, while the collision model predicts the opposite. This suggests that binary disruption may be at work. Extrapolating, we estimate that the share contributed by collisions in lower collision-rate environments is less than $20 \%$, thus demonstrating a more significant contribution from binary coalescence.

Although our results for 24 Magellanic Cloud clusters appear very similar to those published previously for Galactic GCs, we emphasise that the Magellanic Cloud clusters represent a special environment for the study of BSS formation in star clusters. The underlying formation channels might be different in the Magellanic Clouds compared with the Milky Way. Indeed, the low stellar densities and low collision rates in the Magellanic Clouds provides a great opportunity to resolve the tension between observations and simulations in the interpretation of the observed correlation between BSS number and core mass in Galactic GCs. 


\section{Acknowledgements}

RdG would like to thank the organisers of the conference 'Instability Phenomena and Evolution of the Universe' (September 2018), held in honour of Viktor Ambartsumian's 110th birthday, for their invitation to deliver this talk as well as for their partial financial support. This work was supported by the National Key Research and Development Program of China through grant 2017YFA0402702 (RdG). We also acknowledge research support from the National Natural Science Foundation of China (grants U1631102, 11373010, and 11633005). CL is supported by the Macquarie Research Fellowship Scheme.

\section{References}

Bressan, A., Marigo, P., Girardi, L., et al. 2012, MNRAS, 427, 12

Chatterjee, S., Rasio, F. A., Sills, A., \& Glebbeek, E. 2013, ApJ, 777, 106

Ferraro, F. R., Beccari, G., Dalessandro, E., et al. 2009, Nat., 462, 1028

Knigge, C., Leigh, N., \& Sills, A. 2009, Nat., 457, 288

Leigh, N., Knigge, C., Sills, A., et al. 2013, MNRAS, 428, 897

Li, C., de Grijs, R., \& Deng, L. 2013, MNRAS, 436, 1497

Li, C., Deng, L., Bekki, K., et al. 2018, AJ, 156, 110

Milone, A. P., Piotto, G., Bedin, L. R., et al. 2012, A\&A, 540, A1

Sandage, A. R. 1953, AJ, 58, 61

Sun, W., Li, C., de Grijs, R., \& Deng, L. 2018, ApJ, 862, 133 